\title{
Towards an Airframe Noise Prediction Methodology: Survey of Current Approaches
}

\author{
F. Farassat and Jay H. Casper
}

\author{
AIAA-2006-0210, Presented at AIAA 44th Aerospace Sciences Meeting and Exhibit, Reno, Nevada, January 9-12, 2006 \\ NASA Langley Research Center, Hampton, Virginia \\ feri.farassat@nasa.gov, Jay.H.Casper@nasa.gov
}

\begin{abstract}
In this paper, we present a critical survey of the current airframe noise (AFN) prediction methodologies. Four methodologies are recognized. These are the fully analytic method, CFD combined with the acoustic analogy, the semi-empirical method and fully numerical method. It is argued that for the immediate need of the aircraft industry, the semi-empirical method based on recent high quality acoustic database is the best available method. The method based on CFD and the Ffowcs WilliamHawkings $(\mathrm{FW}-\mathrm{H})$ equation with penetrable data surface $\left(\mathrm{FW}-\mathrm{H}_{\mathrm{pds}}\right)$ has advanced considerably and much experience has been gained in its use. However, more research is needed in the near future particularly in the area of turbulence simulation. The fully numerical method will take longer to reach maturity. Based on the current trends, it is predicted that this method will eventually develop into the method of choice. Both the turbulence simulation and propagation methods need to develop more for this method to become useful. Nonetheless, the authors propose that the method based on a combination of numerical and analytical techniques, e.g., CFD combined with FW-H equation, should also be worked on. In this effort, the current symbolic algebra software will allow more analytical approaches to be incorporated into AFN prediction methods.
\end{abstract}

\section{1- Introduction}

Airframe noise prediction is a major area of research in aeroacoustics at present. As aircraft noise standards become more stringent, the attention of the aircraft designers has focussed on the airframe noise (AFN) abatement to reduce the aircraft noise impact on the communities near the airport during take off and landing. Currently, the propulsion system noise has been reduced to such an extent that it is of the same order of magnitude as the airframe noise at landing. There are three sources of AFN in an aircraft. These are: i- turbulent flow over and in the vicinity of flap side-edges, ii- the scattering of the energy of the turbulent eddies in the BL of the wings and high lift devices (flaps and slats) passing over their trailing edges, generating the so-called trailing edge (TE) noise, and iii- bluff body vortex shedding of landing gears and struts causing surface pressure fluctuations on these structures as well as fuselage turbulent BL flow over the wheel-well cavities (cavity noise). By an order of magnitude analysis of these sources of AFN, one can show that in the current generation of aircraft, they contribute equally to the overall noise level during take off and landing. Therefore, attention must be paid to the reduction of all these noise sources. By a simple analysis of the acoustic intensity, one can show that all three sources of AFN must be reduced in decibel levels equally to affect the overall sound level by about the same decibel level. Therefore, the prediction of AFN of an aircraft in the design stage becomes imperative. It is desirable and, economically more sensible, to be able to compute the AFN rather than rely on expensive hardware and experiments to reduce the noise of an aircraft in the design stage. Many significant acoustic experiments at NASA and elsewhere have helped in understanding the physics of noise generation of $\mathrm{AFN}^{1-5}$. These advances as well as the development of computational and analytical tools have made the computation of AFN achievable to a large extent today. It is the aim of this paper to review the available methodologies of AFN prediction and their usefulness and shortcomings.

Airframe noise is broadband in nature and is associated with turbulent flow over aircraft surfaces, trailing edges, landing gears and wheel-wells. For this reason, accurate and reliable AFN prediction is one of the most difficult problems of aeroa- 
coustics $^{1-3}$. In recent years, the technology of turbulence simulation on a computer has advanced significantly. However, simulation of small scale turbulence is still difficult and computationally intensive. Nevertheless, the use of numerical simulations for AFN prediction is becoming more popular as the computation cost goes down. As is shown below, this will lead to better predicted AFN levels.

There are several good reviews of AFN and the challenges facing researchers in predicting this noise ${ }^{6-9}$. One of the most comprehensive reviews is by Crighton ${ }^{6}$. Although written in 1980's, it contains a wealth of useful information on AFN. For example, there is a good review of early works on AFN on model and full scale aircraft with parametric relations for SPL and OASPL derived from measured data. There is a thorough review of the physics and the theory for TE noise prediction as well as the correlation with experimental data. The works of Howe, Amiet, Brooks and Hudgson, Goldstein, and others on TE noise are discussed in detail. Other mechanisms of AFN generation and prediction are also discussed at length. Crighton's review includes an exhaustive and critical analysis of much of the available theories and experimental data of AFN up to the late 1980 's. Other review articles ${ }^{7-9}$ are of more recent vintage and include the experience gained in AFN prediction since the time of Crighton's article. Lockard and Lilley discuss the minimum noise of aircraft of modern aircraft with a detailed order of magnitude analysis of various sources of $\mathrm{AFN}^{7}$. They propose several methods of aircraft noise control such as airport planning and changes in take off and landing procedures.

In the next section, we present the current approaches to AFN prediction. Except for a minority of researchers, combining a near field nonlinear CFD computation with an analytic acoustic propagation technique in various ways, is currently the most common method of AFN prediction. The method based on the acoustic analogy (AA) is by far the most popular method. At present, because of the excessive time taken by turbulence simulation on a computer, and some of the limitations of this simulation, a semi-empirical method appears most attractive for AFN prediction when realistic aircraft designs and operating conditions must be used. However, with the advances in computer technology and numerical methods, one can envision that the future trend will be toward more reliance on turbulence simulation and numerical schemes for noise generation and propagation and less dependence on analytical work. In contrast to this trend, we propose that a noise prediction methodology based on a combination of analytical and numerical methods should also be worked on for AFN prediction because of its inherent efficiency.

Since early 90's, two NASA programs, the Advanced Subsonic Technology (AST) program and the Quiet Aircraft Technology (QAT) program, in cooperation with the industry and academia have resulted in considerable advances in both modeling and prediction of $\mathrm{AFN}^{1}$. Many model and full scale experiments were conducted. The noise prediction effort has been directed toward flow simulation by advanced CFD techniques in the near field combined with an acoustic code for propagation to the far field. Some very significant questions have been answered which can guide the aeroacousticians in improving AFN prediction. We will highlight these achievements in Section 3 below. In Section 4 some theoretical issues in the prediction of AFN are discussed. Finally, in Section 5, Concluding Remarks, some recommendations concerning future work on AFN prediction are presented.

\section{2- Review of Current AFN Prediction Methodologies}

We are interested in prediction of commercial aircraft airframe noise primarily during landing. Specifically, we are interested in quantifying AFN precisely and to compute its contribution to the overall noise of the aircraft. Before we present a review of current AFN prediction methodologies, we point out the desired characteristics of a good noise prediction method. These characteristics are that the AFN prediction methodology must be capable of dealing with:

i- arbitrary aircraft geometry, particularly the flaps, slats and other control surfaces,

ii- arbitrary kinematics of the flight during take off and landing, i.e., speed, glide slope, turns, etc.,

iii- arbitrary observer position, both in the near and far fields, and

additionally, it should have:

iv- quick turnaround time on a computer, i.e., at most a 24 hour turnaround time

No available methodology today possesses all the above characteristics. There are several reasons behind this, chief among them is the inability to predict the loading associated with turbulence on aircraft surfaces and trailing edges. We are mention- 
ing these here because they can be used as one measure of evaluating an AFN prediction methodology. We will now present a survey of AFN prediction methodologies.

The terminology of the hybrid method or technique is often used in the literature of AFN noise prediction. By a hybrid method, we mean combining two different computational techniques, e.g., CFD in the near field to find the source strengths and the acoustic analogy for propagation of sound to the far field, in such a way that the combined methodology is computationally very efficient ${ }^{10}$. The hybrid methods are often a natural choice because engineers and scientists are always in search of speeding up computations. In fact, all available helicopter and propeller noise prediction codes at NASA are hybrid codes 11,12 . By this definition, most of the methodologies discussed below are hybrid techniques, even the fully numerical methods. A survey of AFN prediction methodologies will follow. The next section will present some of the results relevant to the discussions in this section.

\section{1- The Fully Analytical Method}

In this method both the fluid mechanic and acoustic results used in the analysis are obtained analytically. The fully analytical method is generally suitable for studying relatively simple models at the level of basic research. As all researchers know, to understand the physics of a phenomenon, one must start with as simple a model as possible which retains some of the physics of the phenomenon. The works of Ffowcs Williams and Hall ${ }^{13}$, and Crighton ${ }^{14}$ fall in this category. Ffowcs Williams and Hall modeled TE noise generation by the passage of a quadrupole in the vicinity of the edge of a half-plane. Crighton modeled TE noise by a passage of a vortex around the edge of a half-plane. Almost all of Howe's work falls into this category ${ }^{15-17}$. Amiet's works are also generally fully analytical ${ }^{18-20}$. Howe and Amiet are able to obtain significant results by appropriate approximations in geometry, turbulence model, observer position, etc. This method is very valuable to engineers and designers because one is able to obtain $6,15-17,18-20$ :

i- the dependence of the noise intensity on the parameters of the problem, e.g., power laws

ii- scaling laws for design of experiments

iii- rules of thumb for noise reduction, e.g., by changing the path of turbulence eddies near edges

iv- knowledge of radiation directivity pattern

$\mathrm{v}$ - the basis for developing a semi-empirical method (see Subsection 2.3)

On the other hand, the fully analytical method has the following shortcomings:

i- the model could be too simple to represent the real situation, e.g., see the Crighton's model of TE noise ${ }^{14}$

ii- there may be restrictions on geometry, surface motion and observer position of the model

In general, when combined with the use of computers, this method is highly efficient ${ }^{21,22}$. One would expect that the fully analytic method to be very popular. Unfortunately this method, as important as it is, is falling out of favor. The AFN problems are very difficult to solve analytically because they are multidisciplinary, requiring deep knowledge of fluid dynamics, acoustics, and advanced mathematics. Today, in the universities, the teaching of advanced mathematics has been superseded by the teaching of numerical methods because of the perceived usefulness of numerical methods in a broad range of applications. Additionally, the literature of the field of aeroacoustics is very large and expanding at a fast rate, making it hard for the young researchers to break into the field. These factors contribute to the present lack of interest in this method by aeroacousticians.

There is some good news that may change the lack of interest in fully analytical methods in the future. The development of symbolic algebra software such as Maple and Mathematica makes is easy to obtain analytic results and develop efficient acoustic codes. Symbolic software can also be used as an aid in analytic research like an experimental tool to test turbulence and acoustic models quickly and with ease. In particular, obtaining the exact or tailored Green's function ${ }^{23,24}$ can be facilitated by using symbolic algebra software. The use of this powerful tool in aeroacoustics research should be explored. One can envision an entirely novel research style in aeroacoustics based on this tool. 
In connection with this method, Farassat and Posey have emphasized the importance of recognizing when to stop making approximations in geometry, turbulence model and kinematics in the analytical work solely for the purpose of getting simple laws or rules in closed form ${ }^{25}$. Though these laws and rules are important for understanding various noise generation phenomena, one should keep in mind that real noise generation problems of commercial aircraft are too complicated to be described by simple laws and rules. A good example of the effective use of analytic methods is given in the next section. The AA based method allows for arbitrary geometries and kinematics while being fully analytic. However, one needs to use the computer to evaluate surface and volume integrals to obtain the noise level. One can say that it is this philosophy of the use of the analytic method that accounts for the current success of the acoustic analogy in a wide range of applications.

\section{2- CFD Combined With The Acoustic Analogy ( $\left.\mathrm{FW}-\mathrm{H}_{\mathrm{pds}}\right)$}

This is by far the most popular methodology of AFN prediction today $7-10$. Singer, Lockard and Lilley consider this as the only hybrid method ${ }^{10}$. This is debatable as mentioned above. In this method, one performs fluid dynamic calculations which often involve turbulent simulation in the near field. The results are then used in an acoustic code which is typically based on the acoustic analogy. The governing equation for this purpose is by Ffowcs Williams and Hawkings 26 . Currently, the most popular method is taking a data surface across which the fluid is allowed to pass, the so called the $F W$ - $H$ equation with penetrable data surface 27 . The penetrable data surface is also called permeable data surface by some authors. We will henceforth denote this style of using AA as $\mathrm{FW}-\mathrm{H}_{\mathrm{pds}}$. See Section 3, item 2, below for some recent history.

A brief discussion of $\mathrm{FW}-\mathrm{H}_{\mathrm{pds}}$ and one of its most useful solutions follow. The article by Farassat on generalized functions 28 gives a good background. Assume that the penetrable data surface in motion is described by $f(\boldsymbol{x}, t)=0$, and it is defined such that $\nabla f=\boldsymbol{n}$ where $\boldsymbol{n}$ is the outward unit normal to the body surface (this implies that $f>0$ outside this surface). One can show that this can always be done. From this definition, it follows that $\partial f / \partial t=-v_{n}$, where $v_{n}$ is the local normal velocity of the surface $f=0$ which is independent of fluid velocity. Assume that $\boldsymbol{u}$ is the fluid velocity, $c$ and $\rho_{0}$ are speed of sound and density in the undisturbed medium, respectively, $T_{i j}$ is the Lighthill stress tensor and $P_{i j}$ is the compressive stress tensor. Then $\mathrm{FW}-\mathrm{H}_{\mathrm{pds}}$ is

$\square^{2} c_{0}^{2} \rho^{\prime} \equiv \square^{2} p^{\prime}=\frac{\partial}{\partial t}\left[\rho_{0} U_{n} \delta(f)\right]-\frac{\partial}{\partial x_{i}}\left[L_{i} \delta(f)\right]+\frac{\partial^{2}}{\partial x_{i} \partial x_{j}}\left[T_{i j} H(f)\right]$

where $\delta(f)$ and $H(f)$ are the Dirac delta function and Heaviside function, respectively. Also:

$$
\begin{aligned}
& U_{n}=\left(1-\frac{\rho}{\rho_{0}}\right) v_{n}+\frac{\rho u_{n}}{\rho_{0}} \\
& L_{i}=P_{i j} n_{j}+\rho u_{i}\left(u_{n}-v_{n}\right)
\end{aligned}
$$

The philosophy behind using $\mathrm{FW}-\mathrm{H}_{\mathrm{pds}}$ is to locate the data surface $f=0$ around a moving noise generating surface in such a way that most or all the noise producing quadrupoles are included within this surface and no volume integration of the quadrupoles outside the data surface would be needed. The data surface must be located in the region of high numerical accuracy. In AFN applications, a large amount of computer time is taken by turbulence simulation $2,3,7-10$. Therefore, to reduce computation cost, the data surface or overall flow simulation region should be made as small as possible.

There is a closed form solution of eq. (1) by Farassat for arbitrary motion of the surface known as Formulation $1 \mathrm{~A}^{29,30}$. In fact, the notations of eqs. (2) and (3) were introduced in an interesting article by di Francescantonio so that he could use Formulation $1 \mathrm{~A}$ in $\mathrm{FW}-\mathrm{H}_{\mathrm{pds}}$ in the far field ${ }^{31}$. Originally, Formulation 1A was developed by Farassat for an impenetrable data surface, e.g., a helicopter rotor or a propeller blade, as intended by Ffowcs Williams and Hawkings 26 . See Section 3 , item 2 , below for some recent history. 
For AFN applications, the main interest is for uniform and rectilinear flight of the aircraft during take off and landing. To get the solution of the $\mathrm{FW}-\mathrm{H}_{\mathrm{pds}}$ with surface sources at uniform rectilinear motion only, one can use the classical solutions of the linear wave equation for surface sources of monopole and dipole type ${ }^{32}$. Such solutions are sometimes used in place of Formulation 1A with the claim that no retarded time calculations are needed (because of a geometric construction called the Garrick Triangle ${ }^{32-34}$ ) and, therefore, they are more efficient than Formulation 1A. For the purpose of discussion below, we call these solutions "Classical Solutions". The Classical Solutions have the following shortcoming:

i- For arbitrary position of the observer inside the data surface, the acoustic pressure from Formulation 1A should vanish if both the input data are specified correctly and the coding of this formulation is performed correctly ${ }^{35,36}$. This very strict test is one of the strongest reasons to use Formulation 1A instead of the Classical Solutions for AFN prediction.

ii- The Classical Solutions are limited to the observer moving with the aircraft. Formulation 1A can be used for both stationary observer as well as an observer moving with the aircraft. This makes it easy to calculate the noise footprint,

iii- Formulation 1A is not limited to uniform rectilinear motion of the aircraft as the Classical Solutions are. In the future, one may require AFN prediction for accelerating or turning aircraft.

iv- The Garrick Triangle construction can also be used in Formulation 1A for retarded time computation for a surface at uniform rectilinear motion $33,34,37$. Therefore, this claimed advantage of the Classical Solutions over Formulation 1A is invalid.

This is the only AFN noise prediction methodology that possesses most of the desired characteristics of a good methodology discussed at the beginning of this section. It is the use of the $\mathrm{FW}-\mathrm{H}_{\mathrm{pds}}$ that gives this method such a great advantage in the propagation part. The main obstacle in the development of this method is again turbulence simulation.

Currently, there is considerable experience in the use of $\mathrm{FW}-\mathrm{H}_{\mathrm{pds}}$ at NASA and elsewhere. Much of the effort has gone into turbulence simulation using various models such as LES and DNS ${ }^{38}$. Much guidance for the use of the this method has come from studying model problems ${ }^{39-41}$. Many technical papers have been published in Europe and the U.S. on FW$\mathrm{H}_{\text {pds }}$. Two interesting recent works are by Tang 42 and Casalino 43 .

It must be mentioned that the FW-H equation can be solved in both time and frequency domains $33,37,44$. One interesting advantage of working in the frequency domain is that the far field statistics of the noise can be related to the statistics of the surface pressure fluctuations on the noise generating surface 37,44 .

Some theoretical issues related to this method are discussed in Section 4.

\section{3- Semi-Empirical Method}

Semi-empirical methods are an old and important tool of engineers. The hydrolicians of nineteenth and early twentieth centuries used semi-empirical methods to design viaducts, channels and dams. The urgency to get valuable results for design and for meeting noise standards has led to development of noise prediction codes which are based on extremely efficient semi-empirical prediction methods 6,45,46. The Aircraft Noise Prediction Program (ANOPP) of NASA Langley depends heavily on the semi-empirical method for predicting various aircraft noise generation mechanisms 6,12 . Brooks and Burley have proposed a semi-empirical method for predicting the broadband noise from blade-wake interaction of helicopter rotor blades 47,48 . Their method is based on the measured blade response to wake turbulence and an extensive aeroacoustic database. Blade response coherence functions were found and used in a noise radiation formula developed by Amiet ${ }^{18}$. This work has direct application to AFN prediction. In a more recent work, Brooks and Humphreys ${ }^{4}$ have used experimental surface pressure and Howe's theory ${ }^{15}$ to predict the flap edge noise with some success. This is obviously a semi-empirical method.

In recent years, a vast amount of high quality acoustic data has been collected by phase array microphones from models and full scale aircraft. Recently, Guo, Yamamoto and Stoker have developed a semi-empirical method for prediction of noise 
from high lift systems ${ }^{49}$. In this work, a large database of airframe noise tests from different aircraft with a range of operating conditions is utilized to develop a model for noise prediction. In addition to gross aircraft parameters, e.g., the dimensions of the high-lift system and flight Mach number, other parameters of noise generation are also included. Based on new measured data, this work uses a new classification of noise sources compared to the old semi-empirical methods. For example, flap side-edge noise is emphasized more than TE noise as one of the dominant AFN components. Generally good agreement between predicted and measured flight data is obtained by this method ${ }^{49}$. There are indications that this semiempirical method is capable of further improvements.

Because of the fact that the other AFN prediction methodologies discussed in this paper have not yet achieved a fully satisfactory state, the semi-empirical method appears to be the best choice for aircraft designers in estimating AFN of an aircraft to meet the community noise standards. Note, however, that this methodology cannot handle the effects of the geometry of the wings and high-lift devices in AFN. In addition, flight conditions other than those in the standard practice are not allowed. These shortcomings will not allow this method to be used for an aircraft of unconventional design or operating conditions. Therefore, this methodology will have to be replaced eventually by superior ones.

\section{4- Fully Numerical Method}

The users of this method advocate the use of fully numerical method for both turbulence simulation and acoustic propagation. Although one can use the Navier-Stokes equation for both flow simulation and propagation, the governing equation for noise propagation is commonly the linearized Euler equation 50 . The proper transfer of source information from near to far field calculation is an active area of research. In the propagation part of the computation, one should reduce the numerical dispersion and dissipation to obtain meaningful results. Tam has proposed a numerical scheme specifically for this purpo$\mathrm{se}^{51,52}$. Other works on this problem are by $\mathrm{Hu}$ et al. ${ }^{53}$, and Bogey and Bailly ${ }^{54}$. Additionally, one must use absorbing and nonreflective boundary conditions at the outer boundaries of computational domain to reduce contamination of the computed acoustic data ${ }^{55}$. There are, however, some serious problems with this method. The frequencies generated by AFN and other sources of an aircraft are so large that a fully numerical method for propagation is not feasible with modern computers when the full physics of the problem are to be included. For this reason, NASA uses codes based on analytical and semi-empirical methods for the long range propagation where the temperature, wind and density profiles of atmosphere as well as a number of energy absorption processes responsible for attenuation of sound are taken into account. On the other hand, if one is interested in a small range of frequencies, or if the nonlinear steepening of the acoustic waves is required, the fully numerical method is a good choice.

Today, this method is far from having all the desired characteristics of an AFN prediction methodology. This is not to say that it will never reach that stage. On the contrary, because of the training of the young engineers and scientists entering the field of aeroacoustics, the trend appears to be in the direction of development of fully numerical methodology.

At present, the use of this method is justified when one wants to study the effect of scattering of AFN and propulsion system noise by the wings and fuselage of the aircraft 56,57 . Typically in these studies, the incident acoustic source, usually from the propulsion system, is specified. The scattering effect can be significant on the noise footprint particularly if the engines are shielded with the specific aim of reducing the community impact of the noise, e.g., as in the blended wing design. In this connection, we mention the work at Langley on Fast Scattering Code (FSC) 58,59 . This work is based on an analytic solution of the Helmholtz equation in the frame moving with the aircraft. Time dependent aircraft noise foot print can be computed also.

\section{3-The Contribution of Langley Researchers}

We will highlight some of the contributions of NASA Langley researchers which have helped in improvements in AFN prediction. Langley engineers and scientists have worked on aeroacoustics for many years both theoretically and experimentally. The following discussion is directly relevant to AFN prediction.

1- One of the significant results for TE noise prediction is that Brooks and Hudgson showed that the knowledge of the loading on and near the TE of an airfoil, can give the correct acoustic radiation level and directivity pattern of TE noise using 
the acoustic analogy 60 . Airfoils with blunt and sharp trailing edges were used in this study. It is important to realize that this result applies to airfoils in a low Mach number stream. Another important result of this paper is the complex nature of TE noise generation. These authors indicated the need for understanding the mechanism of noise generation and the behavior of the turbulent eddies in the boundary layer in the vicinity of the TE. Research on this subject continued with the experimental and analytical works of Brooks and coworkers 4,60 , Amiet and coworkers ${ }^{18-22}$ as well as analytical works of Howe ${ }^{15-17}$.

2- Brentner and Farassat have shown that the use of $\mathrm{FW}-\mathrm{H}_{\mathrm{pds}}$ with the data surface in the near field is an efficient way of including the noise generating quadrupole sources in the flow region around a surface such as a LE slat ${ }^{27}$. This is an important result which has popularized the use of $\mathrm{FW}-\mathrm{H}_{\mathrm{pds}}$ around the world. It was shown by these researchers that the data surface for the Kirchhoff formula ${ }^{35,36}$ must be located in the linear region to give good acoustic radiation result and can give very high levels of sound in the near field that is not correct. Somewhat earlier, di Francescantonio showed the equivalence of the Kirchhoff formula and $\mathrm{FW}-\mathrm{H}_{\mathrm{pds}}$ in the far field where the propagation is linear ${ }^{31}$. This result is not surprising in view of the fact that Brentner and Farassat showed that in this region the difference between source terms of the governing equation of Kirchhoff formula 35,36 and $\mathrm{FW}-\mathrm{H}_{\text {pds }}$ is composed of small second order terms ${ }^{27}$. The use of the $\mathrm{FW}-\mathrm{H}_{\text {pds }}$ was originally proposed by Ffowcs Williams himself 61 .

3- In a significant work by Singer, Brentner, Lockard and Lilley ${ }^{39}$, the FW- $\mathrm{H}_{\text {pds }}$ was used to verify the TE model of Crighton ${ }^{14}$. The work of Brentner and Farassat about the stable variation of the computed acoustic result with respect to changing the size and position of data surface for $\mathrm{FW}-\mathrm{H}_{\mathrm{pds}}$ was verified by studying the noise from the flow around a cylinder with a wake. The large variation of the acoustic prediction, based on the Kirchhoff formula when the data surface was moved into the near field region, was also verified. In addition, by considering the interaction of the vortices from a small plate near the TE of an airfoil with the TE, it was verified that to get the cardioid shape directivity of the radiated sound, one has to integrate the contribution of the acoustic pressure propagating upstream on the airfoil surface over a considerable distance. This indicates one of the weaknesses of using free space Green's function discussed by Howe 23,24 . There is additional discussion of this problem in the following section.

4- When the data surface is located where the turbulent eddies from the flow around a body intersect the surface, it is known that one can get large variations in predicted noise as the data surface position is changed in the region of intersection with the eddies. Singer and Lockard studied this problem by using a simple model problem in which the acoustic waves from a known source were allowed to propagate through a region of flow with shear ${ }^{40}$. Their conclusion is that if the length scale of region of shear flow is greater than the acoustic wave length, then the acoustic wave is influenced by the shear flow. The implication for the $\mathrm{FW}-\mathrm{H}_{\mathrm{pds}}$ equation is that if the data surface is cut by the wake of a body, the predictions are not reliable in the range of acoustic frequencies for which the wavelengths are smaller than the typical eddy size in the wake.

5- In a paper by Casper et al. a careful study of relative contributions of the surface and volume integrals of FW- $\mathrm{H}_{\text {pds }}$ equation was performed using several different problems ${ }^{41}$. The important conclusions of this study are that:

i- in high-lift systems, the contribution of the quadrupoles, i.e., the volumetric sources, to the overall noise is important relative to surface dipole sources, and

ii- the location of the data surface in the near field is critical in noise calculation which also implies that the accuracy of flow simulation in the near field should be maintained for some distance from the rigid surfaces of the lifting system,

iii- if the data surface is taken on the rigid surfaces of the high lift system, there is the possibility of strong cancellation between the surface and volume integrals in the solution of the FW-H equation.

These conclusions are very important in applications of $\mathrm{FW}-\mathrm{H}_{\mathrm{pds}}$ to $\mathrm{AFN}$ prediction problems. 


\section{4- Some Theoretical Issues in The Application of the Acoustic Analogy}

The solution of FW-H $\mathrm{pds}_{\mathrm{p}}$ is based on the free space Green's function of the wave equation. If one studies the derivation of this equation, it is seen that Ffowcs Williams and Hawkings imbedded the exterior problem of radiation of the noise from a moving surface into a problem of wave propagation in the unbounded three dimensional space. This results in the surface terms of the FW-H equation. It turns out that for the prediction of the discrete frequency noise of the helicopter rotor and propeller blades, the surface source terms of the FW-H equation are the dominant sources of sound. In the prediction of the rotor broadband noise and AFN prediction, quite commonly the surface and volume integrals of the FW-H equation are of the same order of magnitude and of opposite $\operatorname{sign}^{41}$. The remedy for this problem is to use the FW- $\mathrm{H}_{\text {pds }}$. This solution also causes some new problems, e.g., the location of the data surface. If the data surface intersects vortices in the wake, the noise prediction can be sensitive to the location of the data surface.

Howe has recommended another solution to the above problem 23,24 : use a Green's function appropriate to the exterior domain of a noise generating surface, the so-called exact or tailored Green's function. Howe derives several exact Green's functions for various problems with some approximations that allows him to obtain analytic results. Other authors have combined analytic and numerical methods to get the exact Green's function pointwise ${ }^{62}$. It is clear from this work that much effort must go into finding the exact Green's function. It is not at all obvious that finding this exact Green's function will add to the understanding of the physics of AFN generation. The Fast Scattering Code (FSC) of NASA Langley 58,59 can numerically construct the exact Green's function for a multi-element airfoil system very efficiently by the equivalent source method.

Howe $^{23}$ discusses the Curle problem 63 and the solution that Powell gives using the image of quadrupoles reflected in the plane boundary 64 . Howe points out that to predict the noise using the Curle formula, one has to integrate the acoustic pressure over a large portion of the plane boundary. In Powell's formulation, this surface integral is simply equal to the contribution of the image quadrupoles. Howe considers Powell's solution, which is based on the exact Green's function, more appropriate. One can give other examples. Obviously, the exact Green's function will not be useful for predicting the discrete frequency noise of helicopter rotors and propellers. In the case of AFN prediction where the quadrupole sources are important, the exact Green's may offer some advantages over the free space Green's function. One major problem in using the exact Green's function is that one cannot easily interpret the geometric behavior of this function in space and time as one does in the case of the free space Green's function.

\section{5- Concluding Remarks}

Based on the study reported here, we can summarize our conclusions as follows:

1- For the immediate need of the aircraft industry, the semi-empirical method based on the analysis of the recent high quality acoustic data, appears to be the most useful methodology. The development of this method should continue based on high quality model and flight data. This method will have to be replaced in the future because of inherent shortcomings.

2- The methodology based on turbulence simulation in the near field and $\mathrm{FW}-\mathrm{H}_{\text {pds }}$ seems to be showing promise as a good candidate for AFN prediction in the next decade. The main cost of computation is turbulence simulation. Small scale turbulence simulation appears to be a difficult problem in need of further research.

3- The determination of the exact Green's function should be explored further as a competitor to the use of the free space Green's function in FW-H equation. It appears that the exact Green's function does offer some advantages over the free space Green's function for some problems. One needs to find the right problems.

4- The fully numerical approach has not yet reached a mature state. In addition to turbulence simulation, the propagation into a large space and the range of acoustic frequencies of interest in AFN problems are obstacles to the development of this method. This is perhaps a methodology that will take the longest to become useful to the aircraft industry. Based on current trends and the training of the young researchers entering the field of aeroacoustics, it is predicted that many years from now, perhaps in two decades, this method will be the most powerful and popular method of AFN prediction.

5- Against the above trend, the present authors are recommending that the methodology based on a combination of numerical and analytic techniques (Subsection 2.2) should also be considered. The reasons for this recommendation are the proven advantages in efficiency and having all the desired characteristics of a good methodology discussed in Section 2 . New tools 
of symbolic algebra such as Maple and Mathematica will make it possible to solve more realistic models of AFN generation and propagation analytically. This is an area in need of exploration.

\section{7- References}

1- M.G. Macaraeg: Fundamental investigations of airframe noise, AIAA-98-2224, 1998, AIAA/CEAS Aeroacoustics Conference, 4th (19th AIAA Aeroacoustics Conference), Toulouse, France, June 2-4, 1998

2- C.L. Streett, D.P. Lockard, B.A. Singer, M.R. Khorrami, and M.M. Choudhari: In search of physics: The interplay of experiment and computation in airframe noise research; flap-edge noise, AIAA 2003-0979, 41st Aerospace Sciences Meeting \& Exhibit, January 6-9, 2002, Reno, Nevada

3- Mehdi R. Khorrami, Meelan Choudhari, Bart A. Singer, David P. Lockard, and Craig L. Streett: In search of the physics: The interplay of experiment and computation in slat aeroacoustics, AIAA 2003-0980, 41st Aerospace Sciences Meeting \& Exhibit, January 6-9, 2002, Reno, Nevada

4- T. F. Brooks, and William M. Humphreys, Jr., Flap edge aeroacoustic measurements and predictions, AIAA Paper No. 2000-1975, 6th AIAA/CEAS Aeroacoustics Conference June 12-14, 2000 Lahaina, Hawaii

5- Mehdi R. Khorrami and Meelan M. Choudhari: Application of passive porous treatment to slat trailing edge noise, NASA/TM-2003-212416, 2003

6- David G. Crighton: Airframe Noise, in Aeroacoustics of Flight Vehicles, Volume 1, Harvey H. Hubbard (Editor), Acoustical Society of America, 1995

7- David P. Lockard and Geoffrey M. Lilley: The airframe noise reduction challenge, NASA TM-2004-213013, 2004

8- Bart A. Singer and Yueping Guo: Development of computational aeroacoustics tools for airframe noise calculations, International Journal of Computational Fluid Dynamics, 18 (6), 2004, 455-469

9- Meng Wang, Jonathan B. Freund and Sanjiva K. Lele: Computational Prediction of Flow-Generated Sound, Annual Reviews of Fluid Mechanics, 38, 2006, 483-512

10- B. A. Singer, D. P. Lockard and G. M. Lilley: Hybrid acoustic prediction, International Journal of Computers \& Mathematics with Applications, 46, 2003, 647-669

11- Kenneth S. Brentner and F. Farassat: Modeling aerodynamically generated sound of helicopter rotors, Progress in Aerospace Sciences, 39, 2003, 83-120

12- W. E. Zorumski, Aircraft Noise Prediction Program Theoretical Manual, Parts 1 and 2, NASA Technical Memorandum83199-PT-1 and PT-2, National Aeronautics and Space Administration, Langley Research Center, Hampton, VA, 1982

13- J. E. Ffowcs Williams and L. H. Hall. Aerodynamic sound generation by turbulent

flow in the vicinity of a scattering half plane, Journal of Fluid Mechanics, 40, 1970, 657-670

14- D. G. Crighton, Radiation from vortex filament motion near a half-plane, Journal

of Fluid Mechanics, 51, 1972, 357-362

15- M. S. Howe: A review of theory of trailing edge noise, Journal of Sound and Vibration, 61, 1978, 437-465

16- M. S. Howe: Trailing edge noise at low Mach numbers, Journal of Sound and Vibration, 225, 1999, 211-238

17- M. S. Howe: Trailing edge noise at low Mach numbers, Part 2: Attached and separated edge flows, Journal of Sound and Vibration, 234, 2000, 761-775 
18- R. K. Amiet: Acoustic radiation from an airfoil in a turbulent stream, Journal of Sound and Vibration, 41(4), 1975, 407420

19- R. K. Amiet: Noise due to turbulent flow past a trailing edge, Journal of Sound and Vibration,47(3), 1976, 387-393

20- R. K. Amiet: A Note on edge noise theories, Journal of Sound and Vibration, 78(4), 1981, 485-488

21- Robert W. Paterson and Roy K. Amiet: Acoustic radiation and surface pressure characteristics

of an airfoil due to incident turbulence, NASA Contractor Report CR-2733, 1976

22- Robert H. Schlinker and R. K. Amiet: Helicopter rotor trailing edge noise, NASA Contractor report CR-3470, 1981

23- M. S. Howe: Theory of Vortex Sound, Cambridge University Press, 2003

24- M. S. Howe: Edge-source acoustic Green's function for an airfoil of arbitrary chord, with application to trailing-edge noise, Quarterly Journal of Mechanics and Applied Mathematics, 54, 2001, 139-155

25- F. Farassat and J. W. Posey: The Role of Analytical Methods in Computational Aeroacoustics, Aerospace National Simulation Symposium, Tokyo, Japan, June 12-13, 2003, paper available at: http:/techreports.larc.nasa.gov/ltrs/PDF/2003/mtg/NASA-2003-anss-ff.pdf, slides available at: http://techreports.larc.nasa.gov/ltrs/PDF/2003/mtg/NASA-2003anss-ffslides.pdf

26- J. E. Ffowcs Williams and D. L. Hawkings: Sound generated by turbulence and surfaces in arbitrary motion, Philosophical Transactions of the Royal Society, A264, 1969, 321-342

27- Kenneth S. Brentner and F. Farassat: An analytical comparison of the acoustic analogy and Kirchhoff formulation for moving surfaces, AIAA Journal, 36(8), 1998, 1379-138628.

29- F. Farassat, Introduction to Generalized Functions With Applications in Aerodynamics and Aeroacoustics, May 1994 (Corrected April 1996), NASA Technical Paper 3428

29- F. Farassat, G. P. Succi, A review of propeller discrete frequency noise prediction technology with emphasis on two current methods for time domain calculations, 1980, Journal of Sound and Vibration, 71(3), 399-419

30- Kenneth K. Brentner, Prediction of Helicopter Discrete Frequency Rotor Noise- A Computer Program Incorporating Realistic Blade Motions and Advanced Formulation, October 1986, NASA TM 87721

31- P. di Francescantonio: A new boundary integral formulation for the prediction of sound radiation, Journal of Sound and Vibration, 202(4), 1997, 491-509.

32- I. E. Garrick, and Charles E. Watkins: A Theoretical Study of the Effect of Forward Speed on the Free-Space SoundPressure Field Around Propellers, NACA Report 1198, 1954

33- David P. Lockard: A. Comparison of Ffowcs Williams-Hawkings solvers for airframe noise applications, AIAA-20022580, 8th AIAA/CEAS Aeroacoustics Conference \& Exhibit, Breckenridge, Colorado, June 17-19, 2002

34- F. Farassat: Linear acoustic formulas for calculation of rotating blade noise, AIAA Journal, 19(9), 1981, 1122-1130

35- F. Farassat and M. K. Myers: Extension of Kirchhoff's formula to radiation from moving sources, Journal of Sound and Vibration, 123 (3), 1988, 451-460

36- F. Farassat: The Kirchhoff Formulas for Moving Surfaces in Aeroacoustics - The Subsonic and Supersonic Cases, NASA Technical Memorandum 110285, September 1996

37- J. Casper and F. Farassat: A new time domain formulation for broadband noise prediction, International Journal of Aeroacoustics, 1(3), 2002, 207-240

38- Mehdi R. Khorrami, Bart A. Singer and Mert E. Berkman: Time-accurate simulations and acoustic analysis of slat freeshear layer, AIAA-2001-2155, 7th

AIAA/CEAS Aeroacoustics Conference and Exhibit, Maastricht, Netherlands, May 28-30, 2001

39- Bart A. Singer, Kenneth S. Brentner, David P. Lockard, and Geoffrey M. Lilley: Simulation of acoustic scattering from a trailing edge, Journal of Sound and Vibration, 230(3), 2000, 541-560 
40- Bart A. Singer and David P. Lockard: Shear effects in aeroacoustic predictions, Journal of Sound and Vibration, 267 (4), 2003, 961-966

41- Jay H. Casper, David P. Lockard, Mehdi Khorrami and Craig Streett: Investigation of volumetric sources in airframe noise simulations, AIAA-2004-2805, 10th AIAA/CEAS Aeroacoustics Conference , Manchester, United Kingdom, May $10-12,2004$

42- Kefan Tang: Numerical Simulation of Flow-Induced Noise by Means of the Hybrid Method with LES and Aeroacoustic Analogy, Dissertation zur Erlangung des akademischen Grades Doktor-Ingenieur, University of Siegen, Germany

43- D. Casalino: An advanced time approach for acoustic analogy predictions, Journal of Sound and Vibration, 261, 2003, $583-612$

44- Yueping Guo: Airframe noise prediction by acoustic analogy: NASA Contract Informal Report, February 2004

45- Martin R. Fink: Propulsive lift noise, in Aeroacoustics of Flight Vehicles, Volume 1, Harvey H. Hubbard (Editor), Acoustical Society of America, 1995

46- Martin R. Fink: Airframe noise prediction, FAA-RD-77-29, 1977

47- T. F. Brooks and C. L. Burley: Rotor broadband noise prediction with comparison to model data, AIAA Paper 20012210, AIAA/CEAS Aeroacoustics Conference and Exhibit, Maastricht, Netherlands, May 28-30, 2001

48- C. L. Burley and T. F. Brooks, M. A. Marcolini, A. G. Brand and D. A. Conner: Tiltrotor aeroacoustic code (TRAC) predictions and comparisons with measurements, Journal of American Helicopter Society, 45(2), 2000, 80-89

49- Y. P. Guo, K. J. Yamamoto and R.W. Stoker: Component-based empirical model for high-lift system noise prediction, Journal of Aircraft, 40, 2003, 914-922

50- C. Bailly, C. Bogey, and D. Juvé: Computation of the flow noise using source terms in linearized Euler's equations. 2000. AIAA Paper 2000-2047.

51- C. K. W. Tam and Z. Dong: A study of the short wave components in computational acoustics, Journal of Computational Acoustics, 1(1), 1993,1-30

52- Christopher K.W. Tam , Jay C. Webb: Dispersion-relation-preserving finite difference schemes for computational acoustics, Journal of Computational Physics, v.107 n.2, p.262-281, Aug. 1993

53- F. Q. Hu, Y. H. Hussaini, and J. L. Manthey: Low-dissipation and low-dispersion Runge-Kutta schemes for computational acoustics, Journal of Computational Physics, 124, 1996, 177-191

54-Christophe Bogey and Christophe Bailly: A family of low dispersive and low dissipative explicit schemes for flow and noise computations, Journal of Computational Physics, 194(1), 2004, 194 - 214

55- C. K. W. Tam and Z. Dong: Wall boundary condition for high-order finite-difference schemes in computational aeroacoustics. Theoretical and Computational

Fluid Dynamics, 6(6), 1994, 303-322

56- D. Stanescu, J. Xu, M.Y. Hussaini and F. Farassat: Computation of engine noise propagation and scattering off an aircraft, International Journal of Aeroacoustics, 1(4), 2002, 403-420

57- D. Stanescu, M. Y. Hussaini and F. Farassat: Aircraft engine noise scattering from fuselage and wings- a computational approach, Journal of Sound and Vibration, 261(2), 2003, 319-333

58- Mark H. Dunn and Ana F. Tinetti: Aeroacoustic Scattering Via The Equivalent Source Method, AIAA-2004-2937, 10th AIAA/CEAS Aeroacoustics Conference , Manchester, United Kingdom, May 10-12, 2004

59- Ana F. Tinetti and Mark H. Dunn: Aeroacoustic Noise Prediction Using the Fast Scattering Code, AIAA 2005-3061, 11th Aeroacoustics Conference, Monterey, California, May 23-25, 2005

61- D. G. Crighton, A. P. Dowling, J. E. Ffowcs Williams, M. Heckl and F. G. Leppington: Modern Methods in Analytical Acoustics- Lecture Notes, Springer-Verlag, 1992 
62- F. Q. Hu, Y. P. Guo and A. D. Jones: On the computation and application of exact Green's function in acoustic analogy, AIAA 2005-2986, 11th Aeroacoustics Conference, Monterey, California, May 23-25, 2005

63- N. Curle: The influence of solid boundaries upon aerodynamic sound, Proceedings of the Royal Society of London, A231, 1955, 505-514

64- A. Powell: Aerodynamic noise and the plane boundary, Journal of the Acoustical Society of America, 32, 1960, 962-990 